\title{
LABNet: Local Graph Aggregation Network with Class Balanced Loss for Vehicle Re-Identification
}

\author{
Abu Md Niamul Taufique Andreas Savakis \\ Rochester Institute of Technology \\ Rochester, NY, 14623 \\ at7133,andreas.savakis@rit.edu
}

\begin{abstract}
Vehicle re-identification is an important computer vision task where the objective is to identify a specific vehicle among a set of vehicles seen at various viewpoints. Recent methods based on deep learning utilize a global average pooling layer after the backbone feature extractor, however, this ignores any spatial reasoning on the feature map. In this paper, we propose local graph aggregation on the backbone feature map, to learn associations of local information and hence improve feature learning as well as reduce the effects of partial occlusion and background clutter. Our local graph aggregation network considers spatial regions of the feature map as nodes and builds a local neighborhood graph that performs local feature aggregation before the global average pooling layer. We further utilize a batch normalization layer to improve the system effectiveness. Additionally, we introduce a class balanced loss to compensate for the imbalance in the sample distributions found in the most widely used vehicle re-identification datasets. Finally, we evaluate our method in three popular benchmarks and show that our approach outperforms many state-of-the-art methods.
\end{abstract}

\section{Introduction}

Vehicle Re-IDentification (VRID) has significant applications in smart traffic control systems, video surveillance and visual target tracking [34, 40,58]. The objective of VRID is to find a vehicle of interest (probe) within a set of vehicles (gallery set) in a single camera or multi camera settings. Various real world scenarios make the VRID problem challenging such as occlusion, minor appearance differences among different vehicles, illumination variations, pose variations, etc.

In recent years significant progress has been made in VRID with the advancement of deep learning techniques $[13,44,70,27]$ and the introduction of large VRID bench-

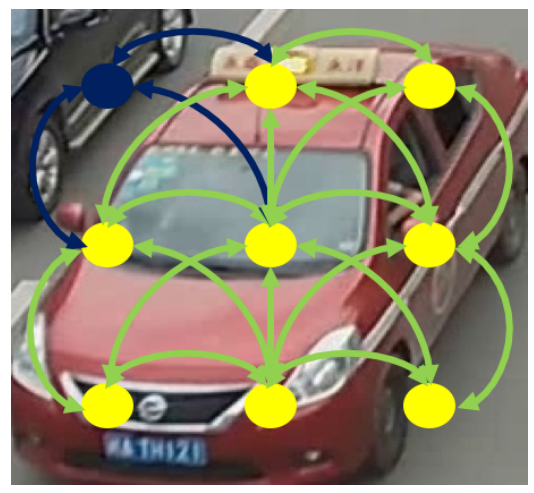

Figure 1: Illustration of spatial information aggregation used by graph network for smoothing spatial features.

marks [40, 37, 34]. In all of these benchmarks, images are captured in a multi-camera settings to get multiple instances of the same vehicle under various pose and illumination conditions. However, in several scenarios, a vehicle might not appear in multiple cameras at the same time or some vehicles are captured more frequently than others. As a result, these benchmarks contain imbalanced sample distributions. In this work, we propose a local graph aggregation network module that associates spatial features (see Fig. 1) and incorporate a class balanced loss to overcome the class imbalance in the training dataset.

Zheng et al [70] showed that the limited number of samples present in several ids may hinder the learning procedure and proposed to use multiple datasets simultaneously to learn a more generalized feature representation. Zheng et al [69] proposed to utilize generative techniques to generate new samples with different viewpoints. The additional training images help achieve pose invariant features and hence better performance. However, both of the aforementioned approaches require additional training data that may be difficult or time consuming to acquire.

Several methods have been proposed to utilize detection and matching of license plate information for VRID [2, 60] 
as the license plate number is unique for each vehicle. However, license plates may not be visible in various circumstances based on the pose of the vehicle or partial occlusion. This suggest that learning visual cues from the vehicle appearance would be key to VRID. The initial works for appearance-based VRID involved hand crafted feature extraction techniques to extract pose and illumination invariant features [35, 66, 25]. Recently, deep learning techniques gained significant popularity due to their superior performance [13, 44, 74, 26].

Despite recent progress, it remains a challenge to learn visual cues that are invariant under intra-class pose, illumination and partial occlusion variations, but also discriminative to the subtle inter-class differences in appearance [44]. Chu et al [8] proposed to tackle the problem of intraclass pose variation using a viewpoint-aware metric learning approach, where they learn two feature spaces for similar and different viewpoints. Meng et al [44] proposed to utilize a network for parsing vehicles into multiple views and then improvise the feature representation based on the parsed mask to align the features for four different viewpoints. Tang et al [56] proposed another method that utilizes synthetic data to estimate vehicle pose and shape information to learn pose invariant features by learning the vehicle type and color information in a multitask learning framework. Chen et al [7] proposed orientation invariant feature learning using semantic guided part attention. He et al [13] proposed to use part detection and attention mechanism to learn fine grained feature. However, all of these methods require extracting additional elements, such as part detection or segmentation, to improve pose invariant properties. Chen et al [6] proposed to split the output feature map of the backbone and learn the spatial significance of the feature map explicitly without any additional annotation of specific parts of the vehicle or keypoints. To achieve this, they partitioned the backbone feature map into multiple partitions and computed losses for these multiple branches. This partition strategy showed improved VRID performance. However, it may suffer due to part missalignment for various poses of the same vehicle [52].

In this research, we proposed a Local graph Aggregation Network with class Balanced loss (LABNet) for VRID. We introduce a local graph aggregation module to learn spatial relationships in the feature map. Local graph aggregation improves pose invariant feature learning under partial occlusion or in complex background scenarios. A simple illustration of our framework is shown in Fig. 1, where a spatial graph is constructed on the extracted backbone feature map (actual constellation is $20 \times 20$ ). Our results demonstrate that stacking graph aggregation modules improves learning under partial occlusion, background clutter and pose variations. We also introduce a class balanced loss along with triplet id loss during network training. The objective is to improve the generalization capabilities of the network by putting more weight on classes with fewer samples. Current methods overlook the class imbalance problem during training, which is known to bias the system towards classes with the largest number of samples. The main contributions of our work are outlined below.

1. We propose a novel parameter-free Local Graph Aggregation (LGA) module after feature extraction for information diffusion among spatial nodes.

2. We present the LABNet architecture for VRID that includes a cascade of LGA modules to increase the receptive field of the feature map and a batch normalization layer for more effective training.

3. We introduce a class balanced loss, that is used in combination with triplet loss for VRID, to tackle the class imbalance problem in the training dataset.

4. Our method is extensively tested and outperforms state-of-the-art methods on several datasets.

The rest of the paper is organized as follows. In Section 2 , we discuss related works. In section 3, we present the details of our method. In section 4, we discuss various VRID benchmarks used for testing and present the experimental details. In section 5, we discuss the evaluation metrics we use to quantify VRID performance. In section 6, we show qualitative results and compare our method to state-of-theart methods. Finally, in section 7, we present final remarks.

\section{Related Work}

Several VRID methods that utilize deep learning techniques have been proposed in recent years. Bai et al [3] proposed a group-sensitive triplet embedding technique to efficiently train deep learning algorithms where the intra-class variance is modeled with an intermediate representation group. Kuma et al [29] compared various sampling techniques during the triplet loss calculation and showed that utilizing proper sampling technique for triplet loss along with id loss can significantly improve VRID performance. He et al [14] proposed to use a multi-domain learning and identity mining to improve the VRID performance. This approach adopted a baseline method [42] that uses soft margin triplet loss and id loss during training. P. Khorramshahi et al [27] used a self-supervised residual generation technique as an attention mechanism to perform VRID. Porrello et al [47] proposed a teacher-student framework and multi-view knowledge distillation to tackle the viewpoint variation in VRID.

\subsection{Feature aggregation using graph networks}

Recently, various research efforts utilize graph based reasoning for VRID and person re-identification (PRID). 


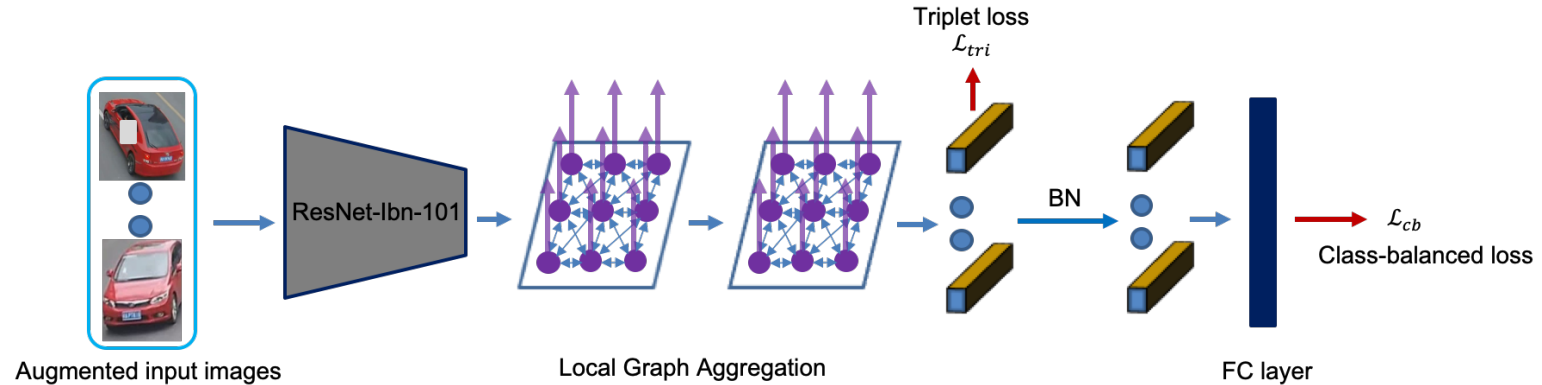

Figure 2: Proposed network architecture. Best viewed in color.

The Pyramidal Graph Network (PGN) [52] is a multiscale pyramidal graph network to incorporate multiscale information from backbone features. The multiscale pyramid is built utilizing various spatial pooling layers and using a Graph Convolution Network (GCN) to diffuse information within the pooled feature maps. Finally, the feature maps from these stages are summed and passed to the classifier to compute the classification score. The overall graph-based information aggregation shows improved performance over the baseline. The Masked Graph Attention Network (MGAT) [4] utilized graph based information processing on a minibatch of images, where features of each image are considered as a node and their mutual information is processed to learn an updated representation for PRID.

Another approach for aggregating features is to use partlevel vehicle information. For part-level information aggregation, horizontal or vertical parts of the backbone feature map are aggregated and the loss is computed for all the part-level features [49]. This type of feature aggregation helps with pose invariant and fine grained feature learning for VRID. In this work, we consider local neighborhood graph aggregation for explicit spatial reasoning. We also utilize multiple graph modules that are stacked to accumulate information within a larger local region.

\subsection{Loss functions}

In various VRID tasks, adding multiple loss functions generally helps to achieve improved performance. The most commonly used loss function is the id loss, or softmax loss function [55], that achieves better convergence. Label smoothing is another component that is mostly used in combination with the id loss to alleviate overfitting [27, 52] in VRID tasks. Another popular loss function for VRID is the triplet loss [50]. Several works have utilized both the triplet loss and the id loss to train the VRID algorithms $[27,52,16]$. However, existing VRID methods do not address the dataset imbalance problem with a class balances loss, such as the one introduced in [9].

\subsection{Dataset augmentation and balance}

Learning pose invariant features requires large labeled datasets, as training deep networks on a small datasets often leads to overfitting. However, collecting and annotating large VRID datasets is a time consuming and expensive task. To address these issues, various augmentation techniques are used during training deep learning models for VRID. Popular augmentation techniques such as random cropping, random flipping, rotation and scaling are often used for training VRID models. Additionally, random erasing augmentation [52, 27, 14] shows significant performance gain.

Furthermore, dataset imbalance is a significant issue with VRID, as illustrated by the distribution of ids shown in Fig. 3. The problem of class imbalance is widely studied in machine learning [21], as it often leads to classification bias. We adapt the class balanced loss proposed in [9] that is designed to counteract the effects of dataset imbalance.

\section{Methodology}

The overall architecture of our method is shown in Fig. 2. The input images are passed through the backbone feature extractor and the local graph is built on top of the backbone feature map. Then the aggregated features are used to compute the triplet loss function. A batch normalization (BN) layer is included to improve the effectiveness of training and it is followed by a fully connected (FC) layer. The output of the FC layer is used to compute the class balanced loss.

\subsection{Local graph based feature aggregation}

We consider an input sample $X \in \mathbb{R}^{3 \times W \times H}$, where $W$ and $H$ are the image width and height respectively, that is processed through the backbone. The output feature map can be written as $\mathbf{x} \in \mathbb{R}^{c \times w \times h}$ where $c$ is the feature dimensions, $w$ is the width and $h$ is the height of the feature map. The width and height of the feature map depend on the pooling, padding, and stride of the backbone network. We build a graph $G(\mathcal{V}, \mathcal{E})$ with nodes $\mathcal{V} \in \mathbb{R}^{k}$ and edges 
$\mathcal{E} \in \mathbb{R}^{k \times k}$, where $k=w \times h$.

For the edge formation, we assume the output backbone feature map, of width and height $w \times h$, lies in a Euclidean coordinate space as depicted in Fig. 2. For node $i \in \mathcal{V}$ with all possible edges $(j, i) \in \mathcal{E}$ where $j \in \mathcal{V}$, the neighborhood set can be written as follows.

$$
\mathcal{N}(i)=\left\{j \mid d\left(p_{i}, p_{j}\right)<r\right\}, \forall j, j \neq i
$$

where, $p_{i}$ and $p_{j}$ are coordinate locations of node $i$ and $j$ respectively, $d(.,$.$) denotes Euclidean distance, and r$ is the distance threshold for the edge formation. The $n^{t h}$ aggregation layer [10] can be written as follows.

$\mathbf{x}_{i}^{n}=\operatorname{ReLU}\left(\sum_{j \in \mathcal{N}(i) \cup\{i\}} \frac{1}{\sqrt{\operatorname{deg}(i)} \cdot \sqrt{\operatorname{deg}(j)}} \cdot\left(\mathbf{x}_{j}^{(n-1)}\right)\right)$

We consider the graph $G(\mathcal{V}, \mathbf{A})$, where $\mathbf{A} \in \mathbb{R}^{k \times k}$ is an affinity matrix, ane the aggregation function can be written as follows.

$$
\mathbf{x}_{a}=\operatorname{Re} L U\left(\tilde{\mathbf{D}}^{-\frac{1}{2}} \tilde{\mathbf{A}} \tilde{\mathbf{D}} \mathbf{x}\right)
$$

where $\tilde{\mathbf{A}}=\mathbf{A}+\mathbf{I}$ is the affinity matrix with the self loops, $\tilde{\mathbf{D}} \in \mathbb{R}^{k \times k}$ is the degree matrix of $\tilde{\mathbf{A}}$ and $\mathbf{x}_{a}$ is the output aggregated feature and $R e L U$ is the non-linearity operation. The propagation rule is adapted from [28].

The receptive field of the aggregated feature map can be increased by increasing $r$, but that adds significant computational overhead and requires large memory. To overcome this limitation, we add multiple LGA layers in cascade that efficiently increase the receptive field of the feature map. With this arrangement, we can effectively increase the receptive field of the aggregated feature map without adding significant computational overhead. The output of the last LGA layer is propagated through a global average pooling (GAP) layer and directly used for the triplet loss computation. We implemented several types of graph-based feature aggregation techniques and found that parameter-free aggregation yields the best results. We found out that adding a weight matrix during the graph propagation caused overfitting of the model and hurt the final VRID performance. We also considered various graph pooling techniques to combine the outputs of nodes from the backbone feature map but this did not improve performance.

Similar to previous studies [27, 14], we found that utilizing a batch normalization (BN) layer before the $\mathrm{FC}$ layer yields the best performance. We include a BN layer after the final LGA module and before the FC layer as depicted in Fig. 2.

\subsection{Loss functions}

The output of the local graph module is used for the computation of the triplet loss [14] that is constructed as follows.

$$
\mathcal{L}_{\text {tri }}=\log \left[1+\exp \left(\left\|\mathbf{x}^{a}-\mathbf{x}^{p}\right\|_{2}^{2}-\left\|\mathbf{x}^{a}-\mathbf{x}^{n}\right\|_{2}^{2}+m\right)\right]
$$

To tackle the class imbalance problem for id loss, we propose to adapt the class balancing loss proposed in [9]. For an input sample $\mathbf{X}$ the output score of the network with one graph module can be written as follows.

$$
\mathbf{z}=F C(B N(G(B F(\mathbf{X}))))
$$

where, $B F$ is the backbone feature extraction network, $G$ is the local graph aggregation module, $\mathrm{BN}$ is the batch normalization layer and $F C$ is the fully connected layer.

The class balanced id loss [9] can be written as follows.

$$
\mathcal{L}_{C B}(\mathbf{z}, y)=-\frac{1-\beta}{1-\beta^{n_{y}}} \log \frac{\exp \left(\mathbf{z}_{y}\right)}{\sum_{t=1}^{T} \exp \left(\mathbf{z}_{t}\right)}
$$

where $T$ is the number of classes and the ground truth label is $y \in 1,2, \ldots . ., T$. The overall loss function of LABNet is the summation of the class balanced loss and the triplet loss that is given as follows.

$$
\mathcal{L}=\mathcal{L}_{C B}(\mathbf{z}, y)+\mathcal{L}_{t r i}
$$

In Eq. (6), the term multiplying the cross-entropy loss is a smoothing term for class balancing. The hyperparameter $\beta \in[0,1)$ and $n_{y}$ is the class frequency in the entire training set. One interesting property of the weighting term is that with $\beta=0$, there is no re-weighting and with $\beta \rightarrow 1$, the re-weighting is the inverse of class frequency. This property allows the flexibility to tune the $\beta$ parameter and optimize the training procedure for given dataset.

\section{Datasets and Experiments}

\subsection{Datasets}

We use three popular benchmarks for the evaluation of LABNet. Some key comparisons among these datasets are shown in Table 1. In Fig. 3 (a),(b),(c), the dataset distribution is shown for VERI [37], VehicleID [34], and VERIWild [40] respectively. In these figures the id imbalance is clearly visible in all of the three benchmarks. A short description of the benchmarks is provided below.

VeRi776 [37] is one of the most popular benchmarks for vehicle re-identification. VERI consists of 49,325 samples of 776 classes. Among them, 576 classes with 37,746 samples are training samples and the remaining 200 classes with 11,579 samples are for testing. The overall data collection is performed with 20 cameras and six viewpoints. In the testing set, 1678 images of the 200 vehicles are randomly 
(a)

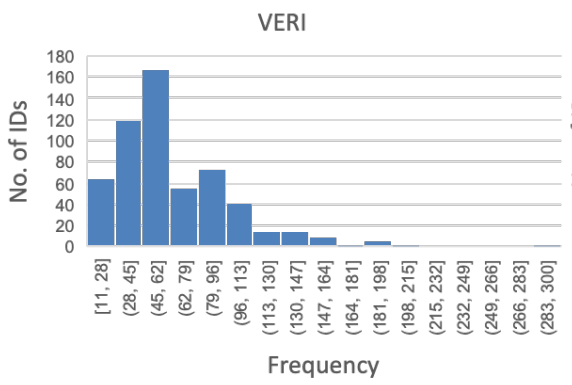

(b)

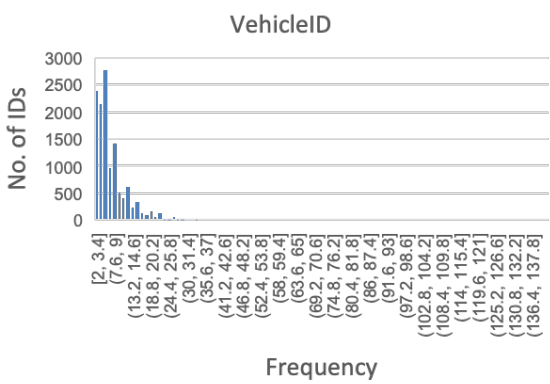

(c)

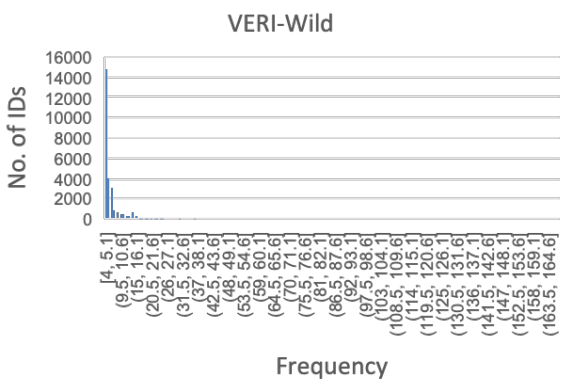

Figure 3: Imbalance in sample distributions for (a) VERI, (b) VehicleID, (c) VERI-Wild benchmarks. Best viewed in color.

Table 1: Dataset description

\begin{tabular}{cc|c|ccc|ccc}
\hline \hline \multicolumn{2}{c|}{ Dataset } & VERI & \multicolumn{3}{c|}{ VehicleID } & \multicolumn{3}{c}{ VERI-Wild } \\
\hline \multirow{2}{*}{ Train } & Images & 37,746 & \multicolumn{3}{c|}{110,178} & \multicolumn{3}{c}{277,797} \\
& Vehicles & 576 & & 13,164 & & 30,671 & \\
\hline \multirow{3}{*}{ Test } & Subset & $\mathrm{X}$ & small & medium & large & small & medium & large \\
& Gallery & 11,579 & 800 & 1,600 & 2,400 & 41,816 & 69,389 & 138,517 \\
& Probe & 1,678 & 5,693 & 11,777 & 17,377 & 3,000 & 5,000 & 10,000 \\
& Vehicles & 200 & 800 & 1,600 & 2,400 & 3,000 & 5,000 & 10,000 \\
\hline \multicolumn{2}{c|}{ Camera } & 20 & \multicolumn{3}{c|}{12} & \multicolumn{3}{c}{174} \\
\hline \multicolumn{2}{c|}{ Views } & 6 & \multicolumn{3}{c}{2} & \multicolumn{3}{c}{ unconstrained } \\
\hline \hline
\end{tabular}

selected as the query set. During inference, gallery images from the same camera as the probe image are discarded.

VehicleID [34] is another standard benchmark for VRID. The dataset contains vehicles with only front or rear viewpoint. It is a large-scale dataset with 221,763 images of 26,267 vehicles. The training set contains 110,178 images of 13,164 vehicles. The testing set is divided into three subsets. The small test subset contains 800 gallery images and 5693 probe images of 800 vehicles. The medium test subset contains 1600 gallery images and 11,777 probe images of 1600 vehicles. The large test subset contains 2400 gallery images and 17,377 probe images of 2400 vehicles. When constructing all the probe and gallery subsets, one image is randomly selected for each vehicle and put into the gallery set. Other images of the same vehicle are considered as the probe images. This procedure is repeated until the desired number of samples are drawn, in a manner similar to the dataset split in the benchmark paper.

Veri-Wild [40] is a benchmark that is widely adopted by the community. It is a large scale dataset that has been collected under different operating conditions, such as morning, afternoon, night, rainy weather, foggy weather etc. In this benchmark, there are 40,671 vehicles with 416,314 image patches. The dataset was collected with 174 cameras in unconstrained traffic conditions. Further, the overall training set contains 30,671 vehicle ids with 277,797 image patches. The testing set is further divided into three subsets, small, medium, and large. The small subset has 3,000 images as probe and 41,816 images as gallery of 3,000 vehicles. The medium subset has 5,000 probe images and 69,389 gallery images of 5,000 vehicles. The large subset has 10,000 probe images and 138,517 gallery images of 10,000 vehicles. The procedure for creating these subsets is to randomly select the vehicles from the test set and randomly select 1 image for the probe image from each of these vehicles, Then the rest of the images of the same vehicle are put in the gallery set.

\subsection{Experiments}

We used PyTorch as our implementation toolbox and PyTorch Geometric [10] for our Graph module implementation. In our experiments, the resnet101-ibn-a [45] was the feature extraction backbone, as it provided superior performance. We adapted some of our experimental settings from [14]. The network was trained with a batch size of 48 and randomidentity sampler during training. The sampler was responsible to randomly select 8 vehicles in each minibatch with 6 samples for each of them. The input image patches were re-scaled to $320 \times 320$ during both training and testing. For augmentation, the probabilities for both random horizontal flip and random erasing were set to 0.5 . The output feature map shape of the backbone is $20 \times 20$ and our 
Table 2: Results on VERI dataset

\begin{tabular}{|c|c|c|}
\hline Method & mAP Rank-1 & Venue \\
\hline LABNet & $84.6 \quad 97.9$ & Proposed \\
\hline SPAN+CPDM[7] & $68.9 \quad 94.0$ & ECCV 2020 \\
\hline SAVER[27] & 79.696 .4 & ECCV 2020 \\
\hline PVEN[44] & 79.595 .6 & CVPR 2020 \\
\hline HPGN[52] & 80.1896 .72 & arXiv 2020 \\
\hline QD-DLF[74] & 61.8388 .50 & IEEE ITS 2020 \\
\hline Appearance+License[15] & 78.0895 .41 & ICIP 2019 \\
\hline SFF+SAtt[32] & 74.1194 .93 & IGCNN 2019 \\
\hline Part Regularization[13] & $74.30 \quad 94.30$ & CVPR 2019 \\
\hline SAN[48] & 72.5093 .30 & arXiv 2019 \\
\hline PAMTRI[56] & 71.8892 .86 & ICCV 2019 \\
\hline MLFN + Triplet[51] & 71.7892 .55 & CVPRW 2019 \\
\hline MTML+Re-ranking[23] & 68.3090 .00 & CVPRW 2019 \\
\hline MRM[46] & 68.5591 .77 & Neurocomputing 2019 \\
\hline DMML[5] & 70.1091 .20 & ICCV 2019 \\
\hline Triplet Embedding[29] & 67.5590 .23 & IJCNN 2019 \\
\hline MOV1+BS[57] & 67.6090 .20 & CVPR 2019 \\
\hline VANet[8] & 66.3489 .78 & ICCV 2019 \\
\hline GRF+GGL[39] & $61.70 \quad 89.40$ & IEEE TIP 2019 \\
\hline ResNet101-AAVER[26] & $61.18 \quad 88.97$ & ICCV 2019 \\
\hline MRM[31] & $71.40 \quad 87.70$ & ICME 2019 \\
\hline Fusion-Net[22] & 62.4087 .31 & IEEE TIP 2019 \\
\hline Mob. VFL-LSTM[1] & $58.08 \quad 87.18$ & ICIP 2019 \\
\hline MGL[64] & 65.0086 .10 & ICIP 2019 \\
\hline EALN[41] & 57.4484 .39 & IEEE TIP 2019 \\
\hline FDA-Net[40] & N/A 49.43 & CVPR 2019 \\
\hline JDRN+Re-ranking[33] & 73.10 N/A & CVPRW 2019 \\
\hline MAD+STR[20] & 61.1189 .27 & ICIP 2018 \\
\hline RAM[38] & 61.5088 .60 & ICME 2018 \\
\hline VAMI[8] & 61.3288 .92 & CVPR 2018 \\
\hline GSTE[3] & 59.47 N/A & IEEE TMM \\
\hline SDC-CNN[75] & 53.4583 .49 & ICPR 2018 \\
\hline PROVID[37] & 53.4281 .56 & IEEE TMM 2018 \\
\hline NuFACT+Plate-SNN[37] & 50.8781 .11 & IEEE TMM 2018 \\
\hline $\mathrm{SCCN}-\mathrm{Ft}+\mathrm{CLBL}-8-\mathrm{Ft}[71]$ & 25.1260 .83 & IEEE TIP 2018 \\
\hline ABLN-Ft-16[72] & 24.9260 .49 & WACV 2018 \\
\hline NuFACT[37] & $48.47 \quad 76.76$ & IEEE TMM 2018 \\
\hline VST Path Proposals[53] & 58.2783 .49 & ICCV 2017 \\
\hline OIFE+ST[59] & 51.4268 .30 & ICCV 2017 \\
\hline DenseNet121[19] & 45.0680 .27 & CVPR 2017 \\
\hline FACT[34] & 18.7552 .21 & ICME 2016 \\
\hline VGG-CNN-M-1024[35] & 12.7644 .10 & CVPR 2016 \\
\hline GoogLeNet[63] & 17.8952 .32 & CVPR 2016 \\
\hline
\end{tabular}

epoch 10. From Epoch 10 to Epoch 39 we kept the learning rate fixed at $10^{-} 2$ and reduced the learning rate to $10^{-3}$ at epoch 40 and kept it fixed until epoch 69. At Epoch 70, we reduced the learning rate to $10^{-4}$ and kept it fixed until epoch 120. For the VehicleID dataset, we used 0.1 times the learning rate used for VERI or VERI-WILD dataset.

We used 0.97 as the class-balanced loss hyperparameter for VERI and VERI-Wild dataset and 0.4 for the VehilceID dataset. We set the value of $r<2$ in all of our experiments. The number of Local Graph modules was set to 2 based on the experiments on all the datasets. For VehicleID results, we evaluated the performance 5 times and showed the average results for both the mAP and Rank-1 metrics. Finally, during inference, after accumulating all the query and the gallery images, we utilized the cosine distance for computing the evaluation metrics.

\section{Evaluation metrics}

We utilize standard metrics to evaluate the proposed method, specifically the mean Average Precision (mAP) and the Cumulative Match Characteristics (CMC).

Mean Average Precision is the primary metric for comparing various re-identification algorithms which can be written as follows [40].

$$
A P=\frac{\sum_{r_{n}=1}^{n} P R\left(r_{n}\right) \times g t\left(r_{n}\right)}{N_{g t}}
$$

where, $r_{n}$ is the rank of a query in a recall list of size $n$, $\operatorname{PR}\left(r_{n}\right)$ is the precision within the $r_{n}$ images and $g t\left(r_{n}\right)$ is the binary value, i.e. 1 if the $r_{n}^{t h}$ image is correct else $0 . N_{g t}$ is the number of vehicles with same id as the query image. The mean Average Precision is the mean of all $A P$ for all the query images.

Cumulative Matching Characterstics (CMC) indicates if the top $k_{n}$ predictions by the network for a query image appear within a gallery list. The CMC at rank $\alpha$ can be written as follows [40].

$$
C M C @ \alpha=\frac{\sum_{q=1}^{Q} g t(q, \alpha)}{Q}
$$

where $Q$ is the total number of query images and $g t(q, \alpha)$ is 1 if the corresponding query image appears within the rank $\alpha$.

\section{Results}

In this section we compare our method with the stateof-the-art methods and demonstrate quantitative results and the qualitative performance of our method.

local graph network is built on top of that. To train our network with VERI and VERI-Wild benchmarks, we started the learning rate at $10^{-3}$ and linearly increased it to $10^{-2}$ in

\subsection{Quantitative results}

We compare our results with many state-of-the-art methods on the aforementioned benchmarks. We used part of the 
Table 3: Results on VehicleID dataset

\begin{tabular}{|c|c|c|c|c|c|c|c|}
\hline \multirow{2}{*}{ Methods } & \multicolumn{2}{|c|}{ small } & \multicolumn{2}{|c|}{ medium } & \multicolumn{2}{|c|}{ large } & \multirow{2}{*}{ References } \\
\hline & mAP & Rank-1 & mAP & Rank-1 & mAP & Rank-1 & \\
\hline LABNet & 89.64 & 84.02 & 86.24 & 80.18 & 83.48 & 77.2 & Proposed \\
\hline SAVER[27] & N/A & 79.9 & N/A & 77.6 & N/A & 75.3 & ECCV 2020 \\
\hline PVEN[44] & N/A & 84.7 & N/A & 80.6 & N/A & 77.8 & CVPR 2020 \\
\hline HPGN[52] & 89.60 & 83.91 & 86.16 & 79.97 & 83.60 & 77.32 & arXiv 2020 \\
\hline QD-DLF[74] & 76.54 & 72.32 & 74.63 & 70.66 & 68.41 & 64.14 & IEEE ITS 2020 \\
\hline Appearance+License[15] & 82.7 & 79.5 & 79.9 & 76.9 & 77.7 & 74.8 & ICIP 2019 \\
\hline MGL[64] & 82.1 & 79.6 & 79.6 & 76.2 & 75.5 & 73.0 & ICIP 2019 \\
\hline Part Regularization[13] & N/A & 78.40 & N/A & 70 & N/A & 74.20 & CVPR 2019 \\
\hline PRN[6] & N/A & 78.92 & N/A & 74.94 & N/A & 71.58 & CVPRW 2019 \\
\hline Triplet Embedding[29] & 86.19 & 78.80 & 81.69 & 73.41 & 78.16 & 69.33 & IJCNN 2019 \\
\hline MRM[31] & 80.02 & 76.64 & 77.32 & 74.20 & 74.02 & 70.86 & Neurocomputing 2019 \\
\hline XG-6-sub-multi [68] & N/A & 76.1 & N/A & 73.1 & N/A & 71.2 & IEEE ITS 2019 \\
\hline GRF+GGL[39] & N/A & 77.1 & N/A & 72.7 & N/A & 70 & IEEE TIP 2019 \\
\hline MSV[61] & 79.3 & 75.1 & 75.4 & 71.8 & 73.3 & 68.7 & ICASSP 2019 \\
\hline DQAL[18] & N/A & 74.74 & N/A & 71.01 & N/A & 68.23 & IEEE TVT \\
\hline EALN[41] & 77.5 & 75.11 & 74.2 & 71.78 & 71.0 & 69.30 & IEEE TIP 2019 \\
\hline Mob.VFL-LSTM[1] & N/A & 73.37 & N/A & 69.52 & N/A & 67.41 & ICIP 2019 \\
\hline ResNet101-AAVER[26] & N/A & 74.69 & N/A & 68.62 & N/A & 63.54 & ICCV 2019 \\
\hline TAMR[12] & N/A & 66.02 & N/A & 62.90 & N/A & 59.69 & IEEE TIP 2019 \\
\hline MLSR[17] & N/A & 65.78 & N/A & 64.24 & N/A & 60.05 & Neurocomputing 2019 \\
\hline RPM[43] & N/A & 65.04 & N/A & 62.55 & N/A & 60.21 & ICMEW 2019 \\
\hline $\mathrm{SFF}+\mathrm{SAtt}[32]$ & N/A & 64.50 & N/A & 59.12 & N/A & 54.41 & IJCNN 2019 \\
\hline FDA-Net[40] & N/A & N/A & 65.33 & 59.84 & 61.84 & 55.53 & CVPR 2019 \\
\hline GSTE[3] & 75.40 & 75.90 & 74.30 & 74.80 & 72.40 & 74.00 & IEEE TMM 2018 \\
\hline RAM[38] & N/A & 75.20 & N/A & 72.3 & N/A & 67.70 & ICME 2018 \\
\hline $\mathrm{C} 2 \mathrm{~F}[11]$ & 63.50 & 61.10 & 60.00 & 56.20 & 53 & 51.40 & AAAI 2018 \\
\hline VAMI[8] & N/A & 63.12 & N/A & 52.87 & N/A & 47.34 & CVPR 2018 \\
\hline SDC-CNN[75] & 63.52 & 56.98 & 57.07 & 50.57 & 49.68 & 42.92 & ICPR 2018 \\
\hline NuFACT[37] & N/A & 48.90 & N/A & 43.64 & N/A & 38.63 & IEEE TMM 2018 \\
\hline MAD+STR[20] & 82.20 & N/A & 75.90 & N/A & 72.80 & N/A & ICIP 2018 \\
\hline PMSM[54] & 64.20 & N/A & 57.20 & N/A & 51.80 & N/A & ICPR 2018 \\
\hline MSVF[24] & N/A & N/A & N/A & N/A & N/A & 46.61 & GCPR 2018 \\
\hline ABLN-32[73] & N/A & 52.63 & N/A & N/A & N/A & N/A & WACV 2018 \\
\hline DJDL[30] & N/A & 72.30 & N/A & 70.80 & N/A & 68.00 & ICIP 2017 \\
\hline Improved Triplet[67] & N/A & 69.90 & N/A & 66.20 & N/A & 63.20 & ICME 2017 \\
\hline DenseNet121[19] & 68.85 & 66.10 & 69.45 & 67.39 & 65.37 & 63.07 & CVPR 2017 \\
\hline MGR[62] & 62.80 & N/A & 62.30 & N/A & 61.23 & N/A & ICCV 2017 \\
\hline OIFE+ST[59] & N/A & N/A & N/A & N/A & N/A & 67.00 & ICCV 2017 \\
\hline DRDL[34] & N/A & 48.91 & N/A & 46.36 & N/A & 40.97 & CVPR 2016 \\
\hline FACT[34] & N/A & 49.53 & N/A & 44.63 & N/A & 39.91 & ICME 2016 \\
\hline
\end{tabular}

tables from [52] for comparing with existing methods.

Table 2 illustrates the remarkable improvement of VRID techniques on the VERI dataset [37] over the years 20162019. Compared to the best method of 2016, performance improved by $39.52 \%$ in $2017,42.75 \%$ in $2018,59.33 \%$ in
2019. Our method outperforms the state-of-the-art methods on the VERI dataset. It achieves $4.6 \%$ gain from the HPGN [52] method on mAP metric and about $1.2 \%$ gain on the Rank-1 rate. LABNet also outperforms SAVER [27] and PVEN [44] by more than 5\% on the mAP metric. 
Table 4: Results on VERI-Wild dataset

\begin{tabular}{lccccccr}
\hline \multirow{2}{*}{ Methods } & \multicolumn{2}{c}{ small } & \multicolumn{2}{c}{ medium } & \multicolumn{2}{c}{ large } & \multirow{2}{*}{ References } \\
& mAP & Rank-1 & mAP & Rank-1 & mAP & Rank-1 & \\
\hline LABNet & 82.6 & 97.2 & 77.4 & 96.3 & 70.0 & 94.5 & Proposed \\
SAVER[27] & 80.9 & 94.5 & 75.3 & 92.7 & 67.7 & 89.5 & ECCV 2020 \\
PVEN[44] & 82.5 & 96.7 & 77.0 & 95.4 & 69.7 & 93.4 & CVPR 2020 \\
HPGN[52] & 80.42 & 91.37 & 75.17 & 88.21 & 65.04 & 82.68 & arXiv 2020 \\
Triplet Embedding[29] & 70.54 & 84.17 & 62.83 & 78.22 & 51.63 & 69.99 & IJCNN 2019 \\
FDA-Net[40] & 35.11 & 64.03 & 29.80 & 57.82 & 22.80 & 49.43 & CVPR 2019 \\
GSTE[3] & 31.42 & 60.46 & 26.18 & 52.12 & 19.50 & 45.36 & IEEE TMM 2018 \\
Unlabelled GAN[76] & 29.86 & 58.06 & 24.71 & 51.58 & 18.23 & 43.63 & ICCV 2017 \\
GoogleNet[63] & 24.27 & 57.16 & 24.15 & 53.16 & 21.53 & 44.61 & CVPR 2015 \\
HDC[65] & 29.14 & 57.10 & 24.76 & 49.64 & 18.30 & 43.97 & ICCV 2017 \\
DRDL[34] & 22.50 & 56.96 & 19.28 & 51.92 & 14.81 & 44.60 & CVPR 2016 \\
Softmax[36] & 26.41 & 53.40 & 22.66 & 46.16 & 17.62 & 37.94 & ECCV 2016 \\
Triplet[50] & 15.69 & 44.67 & 13.34 & 14.34 & 9.93 & 33.46 & CVPR 2015 \\
\hline
\end{tabular}

Table 3 shows the state-of-the-art comparison in the VehicleID [34] dataset. We also show the chronological improvement on VRID performance over 2016-2019. LABNet achieves competitive performance in comparison to state-of-the-art methods. PVEN [44] and HPGN [52] achieved better performance than LABNet by $1.2 \%$ and $0.41 \%$ respectively on Rank- 1 metrics. However, LABNet achieves $3.6 \%$ better performance than SAVER [27] and 4\% better performance than Appearance+License [15] in Rank1 metrics.

Table 4 presents the VRID performance on the VERIWild [40] dataset. Our method achieves state-of-the-art results compared to the existing VRID techniques. LABNet outperforms PVEN [44] by $0.1 \%$ and $0.5 \%$ in the small subset on the mAP and Rank-1 metrics. For the medium test subset, LABNet achieved $1.4 \%$ and $0.9 \%$ gain over PVEN [44]. LABNet also outperforms PVEN [44] on the large test subset by $0.3 \%$ map and $1.1 \%$ Rank- 1 metrics. The results show that LABNet is performing well on a large scale dataset. Since the VERI-Wild dataset is captured with unconstrained pose of the vehicles, our results indicate that LABNet achieves state-of-the-art pose invariant feature generation.

Table 5 shows how each part of our proposed network improves performance on the VERI [37] dataset. First, the baseline itself achieved superior performance due to the backbone, data augmentation techniques, and training procedure. The addition of augmentation with Random Erasing (RE) further improved the mAP by $3.7 \%$. The incorporation of the BN neck improved the mAP by $3.6 \%$. We also evaluated the feature aggregation performance using Local Average Pooling (LAP) instead of graph based aggregation to compare the performance. However, LAP reduced performance by $0.4 \%$ over the baseline on mAP. The Rank- 1
Table 5: Ablation study on VERI dataset

\begin{tabular}{lcc}
\hline \multirow{2}{*}{ Methods } & \multicolumn{2}{c}{ VERI } \\
& mAP & Rank-1 \\
\hline Baseline+RE+BN+LGA+CB & 84.6 & 97.9 \\
Baseline+RE+BN+LGA & 84.6 & 97.3 \\
Baseline+RE+BN+LAP & 83.1 & 96.5 \\
Baseline+RE+BN & 83.5 & 97.2 \\
Baseline+RE & 78.9 & 96.1 \\
Baseline & 75.2 & 94.2 \\
\hline
\end{tabular}

performance was reduced by $0.7 \%$. We then utilized our LGA module in the network and it improved performance by $1.1 \%$ on mAP. With the addition of Class Balanced (CB) loss, we achieved $0.6 \%$ improvement on Rank-1 performance compared to the Baseline+RE+BN+LGA model. Overall, our model improved the performance by $11.4 \%$ on $\mathrm{mAP}$ and $5.7 \%$ on Rank-1 metric compared to the Baseline model. These experiments demonstrate the effectiveness of our method.

Table 6: Varying LGA on VehicleID dataset

\begin{tabular}{ccccccr}
\hline \multirow{2}{*}{ LGA Modules } & \multicolumn{2}{c}{ small } & \multicolumn{2}{c}{ medium } & \multicolumn{2}{c}{ large } \\
& mAP & Rank-1 & mAP & Rank-1 & mAP & Rank-1 \\
\hline 1 & 89.6 & 83.9 & 86.36 & 80.52 & 83.76 & 77.44 \\
$1+2$ & 89.64 & 84.02 & 86.24 & 80.18 & 83.48 & 77.2 \\
$1+2+3$ & 89.46 & 84.0 & 86.08 & 80.06 & 83.84 & 77.56 \\
$1+2+3+4$ & 89.94 & 84.56 & 86.5 & 80.7 & 83.5 & 77.14 \\
$1+2+3+4+5$ & 90.2 & 85.04 & 85.86 & 79.62 & 83.54 & 77.2 \\
\hline
\end{tabular}

Table 6 shows the results of further experiments that evaluate the performance for various depths of graph mod- 


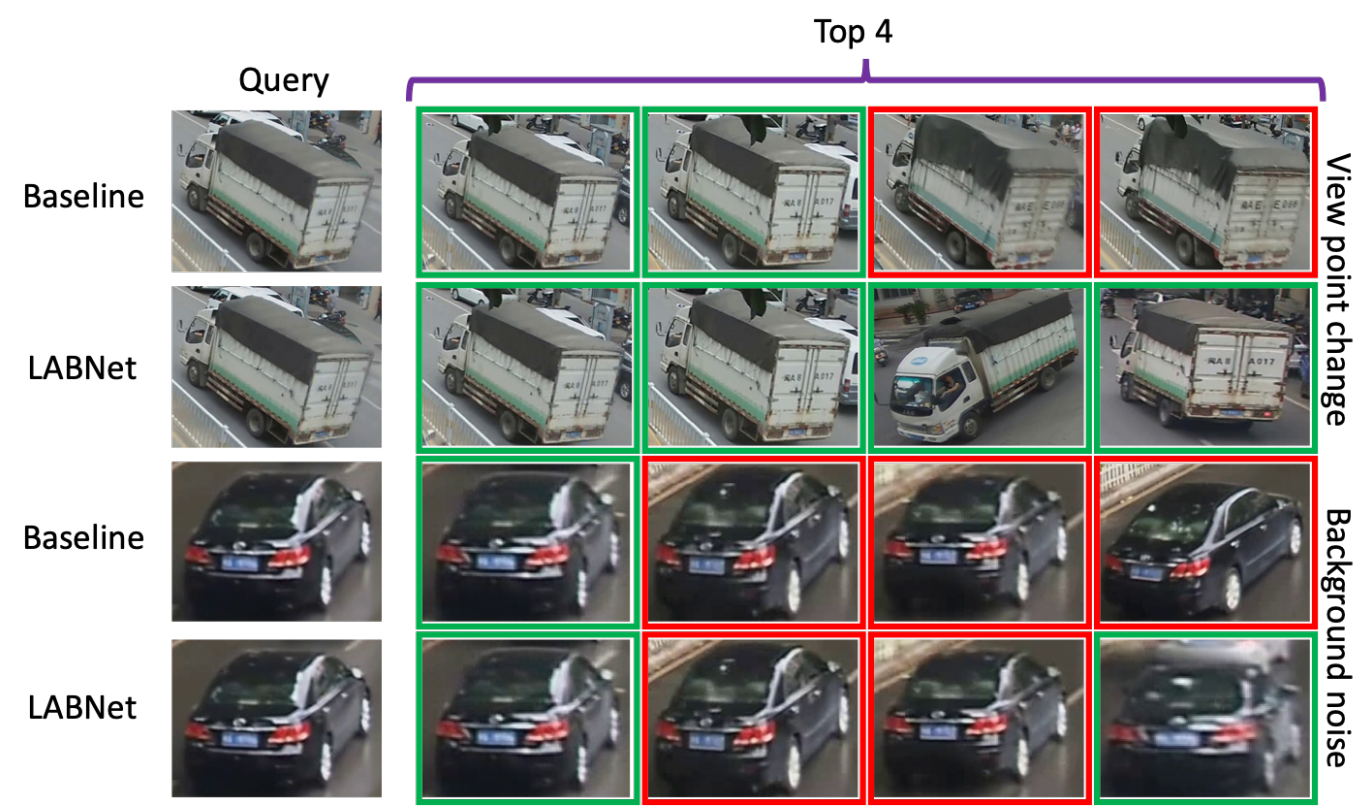

Figure 4: Visual results. Red represents wrong matching and blue represents correct matching. Best viewed in color.

ules on the VehicleID [34] datasets. We found in our experiments that cascading multiple graph modules is beneficial on the small test set. However, the performance on the large test subsets is not affected by the number of LGA modules.

\subsection{Qualitative results and visualization}

Fig. 4 shows two successful cases of the proposed LABNet algorithm, where the top 4 predictions are included with the corresponding query images. For the partial occlusion scenario, LABNet successfully identifies the correct vehicle when the query image or the target images are partially occluded. For the viewpoint change scenario, the baseline method got confused with a vehicle having similar appearance and similar viewpoint. LABNet was able to successfully identify the correct vehicle even though there is slight viewpoint change in the third and fourth position.

\section{Conclusion}

In recent years, deep learning methods achieved significant improvements in the field of vehicle re-identification on various large scale benchmarks. In this paper, we proposed LABNet, a deep learning based method that further advances vehicle re-identification. Our method utilizes graph based feature aggregation on the backbone feature map to aggregate information explicitly among various spatial locations. We incorporate a class balanced loss with the softmax loss during training that improved feature learning on imbalanced datasets. We performed various experiments on multiple benchmarks and our method generally outperforms state-of-the-art methods. Finally, we show qualita- tive results to demonstrate the effectiveness of the proposed method.

\section{Acknowledgments}

This research was supported in part by the Air Force Research Laboratory, Sensors Directorate (AFRL/RYAP) under contract number FA8650-18-C-1739 to Systems and Technology Research. The authors acknowledge the computational resources made available by Research Computing at Rochester Institute of Technology that helped produce part of the results.

\section{References}

[1] S. A. S. Alfasly, Y. Hu, T. Liang, X. Jin, Q. Zhao, and B. Liu. Variational representation learning for vehicle re-identificati. In 2019 IEEE International Conference on Image Processing (ICIP), pages 3118-3122. IEEE, 2019. 6, 7

[2] C.-N. E. Anagnostopoulos, I. E. Anagnostopoulos, I. D. Psoroulas, V. Loumos, and E. Kayafas. License plate recognition from still images and video sequences: A survey. IEEE Transactions on intelligent transportation systems, 9(3):377-391, 2008. 1

[3] Y. Bai, Y. Lou, F. Gao, S. Wang, Y. Wu, and L.-Y. Duan. Group-sensitive triplet embedding for vehicle reidentification. IEEE Transactions on Multimedia, 20(9):2385-2399, 2018. 2, 6, 7, 8

[4] L. Bao, B. Ma, H. Chang, and X. Chen. Masked graph attention network for person re-identification. In Proceedings of the IEEE Conference on Computer Vision and Pattern Recognition Workshops, pages 0-0, 2019. 3 
[5] G. Chen, T. Zhang, J. Lu, and J. Zhou. Deep meta metric learning. In Proceedings of the IEEE International Conference on Computer Vision, pages 9547-9556, 2019. 6

[6] H. Chen, B. Lagadec, and F. Bremond. Partition and reunion: A two-branch neural network for vehicle re-identification. In CVPR Workshops, pages 184-192, 2019. 2, 7

[7] T.-S. Chen, C.-T. Liu, C.-W. Wu, and S.-Y. Chien. Orientation-aware vehicle re-identification with semantics-guided part attention network. arXiv preprint arXiv:2008.11423, 2020. 2, 6

[8] R. Chu, Y. Sun, Y. Li, Z. Liu, C. Zhang, and Y. Wei. Vehicle re-identification with viewpoint-aware metric learning. In Proceedings of the IEEE International Conference on Computer Vision, pages 8282-8291, 2019. 2, 6, 7

[9] Y. Cui, M. Jia, T.-Y. Lin, Y. Song, and S. Belongie. Classbalanced loss based on effective number of samples. In Proceedings of the IEEE Conference on Computer Vision and Pattern Recognition, pages 9268-9277, 2019. 3, 4

[10] M. Fey and J. E. Lenssen. Fast graph representation learning with PyTorch Geometric. In ICLR Workshop on Representation Learning on Graphs and Manifolds, 2019. 4, 5

[11] H. Guo, C. Zhao, Z. Liu, J. Wang, and H. Lu. Learning coarse-to-fine structured feature embedding for vehicle reidentification. In Thirty-Second AAAI Conference on Artificial Intelligence, 2018. 7

[12] H. Guo, K. Zhu, M. Tang, and J. Wang. Two-level attention network with multi-grain ranking loss for vehicle re-identification. IEEE Transactions on Image Processing, 28(9):4328-4338, 2019. 7

[13] B. He, J. Li, Y. Zhao, and Y. Tian. Part-regularized nearduplicate vehicle re-identification. In Proceedings of the IEEE Conference on Computer Vision and Pattern Recognition, pages 3997-4005, 2019. 1, 2, 6, 7

[14] S. He, H. Luo, W. Chen, M. Zhang, Y. Zhang, F. Wang, H. Li, and W. Jiang. Multi-domain learning and identity mining for vehicle re-identification. In Proceedings of the IEEE/CVF Conference on Computer Vision and Pattern Recognition Workshops, pages 582-583, 2020. 2, 3, 4, 5

[15] Y. He, C. Dong, and Y. Wei. Combination of appearance and license plate features for vehicle re-identification. In 2019 IEEE International Conference on Image Processing (ICIP), pages 3108-3112. IEEE, 2019. 6, 7, 8

[16] A. Hermans, L. Beyer, and B. Leibe. In defense of the triplet loss for person re-identification. arXiv preprint arXiv:1703.07737, 2017. 3

[17] J. Hou, H. Zeng, L. Cai, J. Zhu, J. Chen, and K.-K. Ma. Multi-label learning with multi-label smoothing regularization for vehicle re-identification. Neurocomputing, 345:1522, 2019. 7

[18] J. Hou, H. Zeng, J. Zhu, J. Hou, J. Chen, and K.-K. Ma. Deep quadruplet appearance learning for vehicle re-identification. IEEE Transactions on Vehicular Technology, 68(9):85128522, 2019. 7

[19] G. Huang, Z. Liu, L. Van Der Maaten, and K. Q. Weinberger. Densely connected convolutional networks. In Proceedings of the IEEE conference on computer vision and pattern recognition, pages 4700-4708, 2017. 6, 7
[20] N. Jiang, Y. Xu, Z. Zhou, and W. Wu. Multi-attribute driven vehicle re-identification with spatial-temporal re-ranking. In 2018 25th IEEE International Conference on Image Processing (ICIP), pages 858-862. IEEE, 2018. 6, 7

[21] J. M. Johnson and T. M. Khoshgoftaar. Survey on deep learning with class imbalance. Journal of Big Data, 6(1):27, 2019. 3

[22] S. Kan, Y. Cen, Z. He, Z. Zhang, L. Zhang, and Y. Wang. Supervised deep feature embedding with handcrafted feature. IEEE Transactions on Image Processing, 28(12):5809-5823, 2019. 6

[23] A. Kanaci, M. Li, S. Gong, and G. Rajamanoharan. Multitask mutual learning for vehicle re-identification. In Proceedings of the IEEE Conference on Computer Vision and Pattern Recognition Workshops, pages 62-70, 2019. 6

[24] A. Kanac1, X. Zhu, and S. Gong. Vehicle re-identification in context. In German Conference on Pattern Recognition, pages 377-390. Springer, 2018. 7

[25] S. D. Khan and H. Ullah. A survey of advances in visionbased vehicle re-identification. Computer Vision and Image Understanding, 182:50-63, 2019. 2

[26] P. Khorramshahi, A. Kumar, N. Peri, S. S. Rambhatla, J.-C. Chen, and R. Chellappa. A dual-path model with adaptive attention for vehicle re-identification. In Proceedings of the IEEE International Conference on Computer Vision, pages 6132-6141, 2019. 2, 6, 7

[27] P. Khorramshahi, N. Peri, J.-c. Chen, and R. Chellappa. The devil is in the details: Self-supervised attention for vehicle re-identification. arXiv preprint arXiv:2004.06271, 2020. 1, $2,3,4,6,7,8$

[28] T. N. Kipf and M. Welling. Semi-supervised classification with graph convolutional networks. arXiv preprint arXiv:1609.02907, 2016. 4

[29] R. Kuma, E. Weill, F. Aghdasi, and P. Sriram. Vehicle reidentification: an efficient baseline using triplet embedding. In 2019 International Joint Conference on Neural Networks (IJCNN), pages 1-9. IEEE, 2019. 2, 6, 7, 8

[30] Y. Li, Y. Li, H. Yan, and J. Liu. Deep joint discriminative learning for vehicle re-identification and retrieval. In 2017 IEEE International Conference on Image Processing (ICIP), pages 395-399. IEEE, 2017. 7

[31] W. Lin, Y. Li, X. Yang, P. Peng, and J. Xing. Multi-view learning for vehicle re-identification. In 2019 IEEE International Conference on Multimedia and Expo (ICME), pages 832-837. IEEE, 2019. 6, 7

[32] C. Liu, D. Q. Huynh, and M. Reynolds. Urban area vehicle re-identification with self-attention stair feature fusion and temporal bayesian re-ranking. In 2019 International Joint Conference on Neural Networks (IJCNN), pages 1-8. IEEE, 2019. 6, 7

[33] C.-T. Liu, M.-Y. Lee, C.-W. Wu, B.-Y. Chen, T.-S. Chen, Y.T. Hsu, S.-Y. Chien, and N. I. Center. Supervised joint domain learning for vehicle re-identification. In CVPR Workshops, pages 45-52, 2019. 6

[34] H. Liu, Y. Tian, Y. Yang, L. Pang, and T. Huang. Deep relative distance learning: Tell the difference between similar vehicles. In Proceedings of the IEEE Conference on Computer 
Vision and Pattern Recognition, pages 2167-2175, 2016. 1, $4,5,6,7,8,9$

[35] X. Liu, W. Liu, H. Ma, and H. Fu. Large-scale vehicle reidentification in urban surveillance videos. In 2016 IEEE International Conference on Multimedia and Expo (ICME), pages 1-6. IEEE, 2016. 2, 6

[36] X. Liu, W. Liu, T. Mei, and H. Ma. A deep learning-based approach to progressive vehicle re-identification for urban surveillance. In European conference on computer vision, pages 869-884. Springer, 2016. 8

[37] X. Liu, W. Liu, T. Mei, and H. Ma. Provid: Progressive and multimodal vehicle reidentification for large-scale urban surveillance. IEEE Transactions on Multimedia, 20(3):645658, 2017. 1, 4, 6, 7, 8

[38] X. Liu, S. Zhang, Q. Huang, and W. Gao. Ram: a regionaware deep model for vehicle re-identification. In 2018 IEEE International Conference on Multimedia and Expo (ICME), pages 1-6. IEEE, 2018. 6, 7

[39] X. Liu, S. Zhang, X. Wang, R. Hong, and Q. Tian. Groupgroup loss-based global-regional feature learning for vehicle re-identification. IEEE Transactions on Image Processing, 29:2638-2652, 2019. 6, 7

[40] Y. Lou, Y. Bai, J. Liu, S. Wang, and L. Duan. Veri-wild: A large dataset and a new method for vehicle re-identification in the wild. In Proceedings of the IEEE Conference on Computer Vision and Pattern Recognition, pages 3235-3243, 2019. 1, 4, 5, 6, 7, 8

[41] Y. Lou, Y. Bai, J. Liu, S. Wang, and L.-Y. Duan. Embedding adversarial learning for vehicle re-identification. IEEE Transactions on Image Processing, 28(8):3794-3807, 2019. 6, 7

[42] H. Luo, W. Jiang, Y. Gu, F. Liu, X. Liao, S. Lai, and J. Gu. A strong baseline and batch normalization neck for deep person re-identification. IEEE Transactions on Multimedia, pages $1-1,2019.2$

[43] X. Ma, K. Zhu, H. Guo, J. Wang, M. Huang, and Q. Miao. Vehicle re-identification with refined part model. In 2019 IEEE International Conference on Multimedia \& Expo Workshops (ICMEW), pages 603-606. IEEE, 2019. 7

[44] D. Meng, L. Li, X. Liu, Y. Li, S. Yang, Z.-J. Zha, X. Gao, S. Wang, and Q. Huang. Parsing-based view-aware embedding network for vehicle re-identification. In Proceedings of the IEEE/CVF Conference on Computer Vision and Pattern Recognition, pages 7103-7112, 2020. 1, 2, 6, 7, 8

[45] X. Pan, P. Luo, J. Shi, and X. Tang. Two at once: Enhancing learning and generalization capacities via ibn-net. In ECCV, 2018. 5

[46] J. Peng, H. Wang, T. Zhao, and X. Fu. Learning multiregion features for vehicle re-identification with contextbased ranking method. Neurocomputing, 359:427-437, 2019. 6

[47] A. Porrello, L. Bergamini, and S. Calderara. Robust re-identification by multiple views knowledge distillation. arXiv preprint arXiv:2007.04174, 2020. 2

[48] J. Qian, W. Jiang, H. Luo, and H. Yu. Stripe-based and attribute-aware network: A two-branch deep model for vehicle re-identification. Measurement Science and Technology, 2020. 6
[49] R. Quan, X. Dong, Y. Wu, L. Zhu, and Y. Yang. Autoreid: Searching for a part-aware convnet for person reidentification. In Proceedings of the IEEE International Conference on Computer Vision, pages 3750-3759, 2019. 3

[50] F. Schroff, D. Kalenichenko, and J. Philbin. Facenet: A unified embedding for face recognition and clustering. In Proceedings of the IEEE conference on computer vision and pattern recognition, pages 815-823, 2015. 3, 8

[51] A. Shankar, A. Poojary, V. Kollerathu, C. Yeshwanth, S. Reddy, and V. Sudhakaran. Comparative study on various losses for vehicle re-identification. In CVPR Workshops, volume 2, 2019. 6

[52] F. Shen, J. Zhu, X. Zhu, Y. Xie, and J. Huang. Exploring spatial significance via hybrid pyramidal graph network for vehicle re-identification. arXiv preprint arXiv:2005.14684, 2020. 2, 3, 6, 7, 8

[53] Y. Shen, T. Xiao, H. Li, S. Yi, and X. Wang. Learning deep neural networks for vehicle re-id with visual-spatio-temporal path proposals. In Proceedings of the IEEE International Conference on Computer Vision, pages 1900-1909, 2017. 6

[54] Y. Sun, M. Li, and J. Lu. Part-based multi-stream model for vehicle searching. In 2018 24th International Conference on Pattern Recognition (ICPR), pages 1372-1377. IEEE, 2018. 7

[55] Y. Sun, X. Wang, and X. Tang. Deep learning face representation from predicting 10,000 classes. In Proceedings of the IEEE conference on computer vision and pattern recognition, pages 1891-1898, 2014. 3

[56] Z. Tang, M. Naphade, S. Birchfield, J. Tremblay, W. Hodge, R. Kumar, S. Wang, and X. Yang. Pamtri: Pose-aware multitask learning for vehicle re-identification using highly randomized synthetic data. In Proceedings of the IEEE International Conference on Computer Vision, pages 211-220, 2019. 2, 6

[57] Z. Tang, M. Naphade, M.-Y. Liu, X. Yang, S. Birchfield, S. Wang, R. Kumar, D. Anastasiu, and J.-N. Hwang. Cityflow: A city-scale benchmark for multi-target multicamera vehicle tracking and re-identification. In Proceedings of the IEEE Conference on Computer Vision and Pattern Recognition, pages 8797-8806, 2019. 6

[58] A. M. N. Taufique, B. Minnehan, and A. Savakis. Benchmarking deep trackers on aerial videos. Sensors, 20(2):547, 2020. 1

[59] Z. Wang, L. Tang, X. Liu, Z. Yao, S. Yi, J. Shao, J. Yan, S. Wang, H. Li, and X. Wang. Orientation invariant feature embedding and spatial temporal regularization for vehicle re-identification. In Proceedings of the IEEE International Conference on Computer Vision, pages 379-387, 2017. 6, 7

[60] N. Watcharapinchai and S. Rujikietgumjorn. Approximate license plate string matching for vehicle re-identification. In 2017 14th IEEE International Conference on Advanced Video and Signal Based Surveillance (AVSS), pages 1-6. IEEE, 2017. 1

[61] Y. Xu, N. Jiang, L. Zhang, Z. Zhou, and W. Wu. Multi-scale vehicle re-identification using self-adapting label smoothing regularization. In ICASSP 2019-2019 IEEE International Conference on Acoustics, Speech and Signal Processing (ICASSP), pages 2117-2121. IEEE, 2019. 7 
[62] K. Yan, Y. Tian, Y. Wang, W. Zeng, and T. Huang. Exploiting multi-grain ranking constraints for precisely searching visually-similar vehicles. In Proceedings of the IEEE International Conference on Computer Vision, pages 562-570, 2017. 7

[63] L. Yang, P. Luo, C. Change Loy, and X. Tang. A large-scale car dataset for fine-grained categorization and verification. In Proceedings of the IEEE conference on computer vision and pattern recognition, pages 3973-3981, 2015. 6, 8

[64] X. Yang, C. Lang, P. Peng, and J. Xing. Vehicle reidentification by multi-grain learni. In 2019 IEEE International Conference on Image Processing (ICIP), pages 31133117. IEEE, 2019. 6, 7

[65] Y. Yuan, K. Yang, and C. Zhang. Hard-aware deeply cascaded embedding. In Proceedings of the IEEE international conference on computer vision, pages 814-823, 2017. 8

[66] D. Zapletal and A. Herout. Vehicle re-identification for automatic video traffic surveillance. In Proceedings of the IEEE Conference on Computer Vision and Pattern Recognition Workshops, pages 25-31, 2016. 2

[67] Y. Zhang, D. Liu, and Z.-J. Zha. Improving triplet-wise training of convolutional neural network for vehicle reidentification. In 2017 IEEE International Conference on Multimedia and Expo (ICME), pages 1386-1391. IEEE, 2017. 7

[68] Y. Zhao, C. Shen, H. Wang, and S. Chen. Structural analysis of attributes for vehicle re-identification and retrieval. IEEE Transactions on Intelligent Transportation Systems, 21(2):723-734, 2019. 7

[69] Z. Zheng, M. Jiang, Z. Wang, J. Wang, Z. Bai, X. Zhang, X. Yu, X. Tan, Y. Yang, S. Wen, et al. Going beyond real data: A robust visual representation for vehicle reidentification. In Proceedings of the IEEE/CVF Conference on Computer Vision and Pattern Recognition Workshops, pages 598-599, 2020. 1

[70] Z. Zheng, T. Ruan, Y. Wei, Y. Yang, and T. Mei. Vehiclenet: Learning robust visual representation for vehicle reidentification. arXiv preprint arXiv:2004.06305, 2020. 1

[71] Y. Zhou, L. Liu, and L. Shao. Vehicle re-identification by deep hidden multi-view inference. IEEE Transactions on Image Processing, 27(7):3275-3287, 2018. 6

[72] Y. Zhou and L. Shao. Vehicle re-identification by adversarial bi-directional lstm network. In 2018 IEEE Winter Conference on Applications of Computer Vision (WACV), pages 653-662. IEEE, 2018. 6

[73] Y. Zhou and L. Shao. Vehicle re-identification by adversarial bi-directional lstm network. In 2018 IEEE Winter Conference on Applications of Computer Vision (WACV), pages 653-662. IEEE, 2018. 7

[74] J. Zhu, H. Zeng, J. Huang, S. Liao, Z. Lei, C. Cai, and L. Zheng. Vehicle re-identification using quadruple directional deep learning features. IEEE Transactions on Intelligent Transportation Systems, 21(1):410-420, 2019. 2, 6, 7

[75] J. Zhu, H. Zeng, Z. Lei, S. Liao, L. Zheng, and C. Cai. A shortly and densely connected convolutional neural network for vehicle re-identification. In 2018 24th International Con- ference on Pattern Recognition (ICPR), pages 3285-3290. IEEE, 2018. 6, 7

[76] J.-Y. Zhu, T. Park, P. Isola, and A. A. Efros. Unpaired imageto-image translation using cycle-consistent adversarial networks. In Proceedings of the IEEE international conference on computer vision, pages 2223-2232, 2017. 8 